\title{
A Transição das Razões para as Funções Trigonométricas
}

\author{
The Transition from Ratios to Trigonometric Functions
}

\author{
Maria Elisa Esteves Lopes Galvão* \\ Vera Helena Giusti de Souza** \\ Paulo Masanobo Miashiro ${ }^{* * *}$
}

\begin{abstract}
Resumo
Nossa motivação surgiu da constatação de que uma das dificuldades, no estudo das funções trigonométricas, é a compreensão de conceitos como o do ciclo trigonométrico e da medida de um ângulo em radianos. Nosso objetivo foi investigar a contribuição de um ensino apoiado em construções com uma geometria dinâmica e em materiais concretos. Baseados na Teoria da Aprendizagem Significativa, escolhemos os conceitos subsunçores relacionados à definição da função seno e aplicamos uma intervenção, com base no Design Based Research, num trabalho em quatro sessões com nove alunos de uma turma de Licenciatura em Matemática. Para elaborar as atividades, consideramos o desenvolvimento histórico de conceitos da Trigonometria e as recomendações dos Parâmetros Curriculares Nacionais. Ao final da intervenção, verificamos que esta não foi suficiente para uma aprendizagem significativa desses conceitos; contudo, observamos que todos os participantes conseguiram construir uma tabela e um gráfico de uma função periódica e, para dois deles, esse gráfico é o da função seno.
\end{abstract}

Palavras-chave: Aprendizagem Significativa. Conceito Subsunçor. Trigonometria. FunçõesTrigonométricas. Cabri-Géomètre II.

\begin{abstract}
Our motivation comes from the difficulties that appeared in the lessons involving trigonometric functions, related to the trigonometric circle and the measure of an angle in radians. The aim was to investigate the contributions of an approach combining constructions with a dynamic software and concrete material. Based on the Meaningful Learning Theory, we chose subsumed concepts related to the definition of the sinus function and applied a set of activities for nine students from a teachers Course in Mathematics, using the Design Based Research. For drawing up the activities, we considered historical development of concepts of trigonometry and recommendations from the Brazilian National Curriculum Standards. Our intervention was not enough for the participants to achieve a significant learning in trigonometry. However, we observed that everyone managed to build a table and a graph of a periodic function, and two participants were able to sketch the graph of the sinus function.
\end{abstract}

Key Words: Subsumed concept. Trigonometry. Trigonometric functions. Cabri-Géomètre II.

\section{Introdução}

\footnotetext{
* Doutora pela Universidade de São Paulo (USP). Professora da Universidade Anhanguera de São Paulo (UNIAN), São Paulo/SP, Brasil. Endereço para correspondência: Rua Carajuá 60, apto. 12, Moema, CEP: 04520-020, São Paulo/SP, Brasil. e-mail: elisa.gal.meg @ gmail.com

** Doutora pela Pontifícia Universidade Católica de São Paulo (PUC-SP). Professora da Universidade de São Paulo (USP), São Paulo/SP, Brasil. Endereço para correspondência: Rua Edson Bona, 207, Jardim Peri-Peri, CEP: 05538-020, São Paulo/SP, Brasil. e-mail: verahgsouza@gmail.com

**** Mestre pela Universidade Anhanguera de São Paulo (UNIAN). Endereço para correspondência: Rua, $n^{\circ}$, bairro, cidade/UF, Cep: . e-mail: paulo@ miashiro.com
} 
Constatamos, em nossa experiência na atividade docente, as dificuldades enfrentadas pelos estudantes, relacionadas às medidas em radianos, à representação no ciclo trigonométrico e à construção de tabelas trigonométricas e gráficos. Estabelecemos, a partir dessas constatações e de leituras de pesquisas correlatas, o objetivo para a nossa pesquisa: verificar as contribuições de uma estratégia de ensino, formada pela combinação do contexto experimental com o contexto computacional, para a aprendizagem significativa dos principais conceitos presentes na transição das razões no triângulo retângulo para as funções trigonométricas. Com este objetivo em mente, formulamos nossa questão de pesquisa: "Quais as contribuições de uma "estratégia de ensino" para a aprendizagem significativa na transição das razões trigonométricas para as funções trigonométricas?"

Inspirados no trabalho de Brighenti (1994), escolhemos a teoria da Aprendizagem Significativa de Ausubel (AUSUBEL, D.; NOVAK, D. J.; HANESIAN, H., 1978) e criamos um conjunto de atividades introdutórias para, a partir da adaptação de um texto do livro Functions modeling change: a preparation for calculus (CONNALLY; GLEASON; HUGHES-HALLET, 1998), conduzirmos a construção da função seno, orientados pelo percurso histórico de construção dos conhecimentos em Trigonometria. Ao longo de oito encontros de trabalho, com um grupo de nove estudantes de um curso de Licenciatura em Matemática, seguindo a metodologia do Design Experiments (COBB, P. et al, 2003), desenvolvemos atividades nos contextos experimental e computacional, com protocolos em papel e lápis. A análise desses protocolos foi feita com base nas idéias da Teoria dos Registros de Representação Semiótica (DUVAL, 2009), associadas às construções de gráficos de funções, a partir dos dados obtidos em tabelas, com o uso do ciclo trigonométrico e das manipulações com o software CabriGéomètre II. A metodologia adotada permitiu a avaliação e a readequação das atividades durante o trabalho com os participantes.

\section{Alguns aspectos importantes da História da Trigonometria}

Segundo Boyer (1996, p.13), num dos problemas do papiro de Rhind (1650 a.C.), encontram-se vestígios de uma teoria de triângulos semelhantes que deram origem à Trigonometria. De acordo com esse documento, para manterem constantes as inclinações das pirâmides, os egípcios criaram um conceito semelhante ao da cotangente de um ângulo, o "seqt". O seqt da face de uma pirâmide era o quociente do afastamento horizontal pelo vertical. Segundo Eves, (2004, p.95), Thales (640 a.C. - 564 a.C.), filósofo, astrônomo e matemático que viveu em Mileto, na Grécia, calculou a altura de uma pirâmide recorrendo à 
semelhança dos triângulos formados pelas alturas da pirâmide e de uma estaca fincada na areia e suas respectivas sombras.

O movimento dos planetas em torno da Terra, com a figura dos epiciclos, foi descrito por Apolônio (262 a.C.- 190 a.C.), importante matemático e astrônomo, segundo o qual um planeta girava com velocidade uniforme ao longo de um pequeno círculo (epiciclo), cujo centro se movia ao longo de um círculo maior, conhecido como deferente, cujo centro era a Terra. Segundo Maor (1998, p.23), Hiparco, o astrônomo que fez valiosas observações entre 141 e 127 a.C., calculou o movimento dos epiciclos sem contar com tabelas de relações trigonométricas, considerando que, nos triângulos inscritos nos epiciclos, há uma relação entre os lados do triângulo (cordas do epiciclo) e os respectivos ângulos centrais. Por séculos,essa relação entre comprimentos de cordas e ângulos centrais se constituiu a principal tarefa da Trigonometria, motivada pelos estudos da Astronomia ou relacionada a eles. Três séculos depois de Hiparco, no ano 150 d.C., viveu em Alexandria o astrônomo, geógrafo e matemático Klaudius Ptolomaios (ou Ptolomeu) que, de acordo com Aaboe (1997, p.133), é o autor da mais importante obra sobre a Trigonometria na antiguidade, o "Almagesto", um manual de Astronomia, com 13 livros, nos quais ele descreve suas observações sobre fatos astronômicos, considera o sistema geocêntrico para o universo e apresenta uma tábua de cordas, para ângulos de $12^{\circ}$ a $180^{\circ}$, com intervalos de 30', detalhando cada etapa de sua construção. Segundo Katz (2009, p.156), nessa obra, Ptolomeu lançou o germe da moderna ideia de função, apresentando algumas tabelas e exibindo relações funcionais entre conjuntos e quantidades. A "função“ $c r d \alpha$ era definida como sendo o comprimento da corda que correspondia a um arco de $\alpha$ graus em um círculo cujo raio media 60 unidades. Comparada com a função seno, temos que $\operatorname{crd} \alpha=120$. sen $(\alpha / 2)$.

Os hindus aperfeiçoaram os conhecimentos da Trigonometria quando consideraram a relação funcional entre a metade da corda e a metade do ângulo central e, a partir dessa relação,construíram uma tabela para servir de base para os cálculos da Trigonometria esférica. “Assim, aparentemente nasceu na Índia a precursora da função trigonométrica moderna que chamamos de seno de um ângulo, e a introdução da função seno representa a contribuição mais importante dos Sidhantas à história da matemática" (BOYER, 1996, p.143).

Os árabes aperfeiçoaram a álgebra e a trigonometria: o astrônomo Al-Battania adotou a razão jiva dos hindus, teve a genial ideia de introduzir o raio unitário e demonstrou que essa razão é válida para qualquer triângulo retângulo.

No período do Renascimento, surgiram as primeiras publicações específicas, relacionadas à trigonometria, com duas figuras notáveis, Regiomontanus e Copérnico. 
Regiomontanus aprendeu o grego para ler Ptolomeu no original e em 1464 escreveu $D e$ Trianguleis Omnimodis (Os triângulos de todas as espécies), obra dedicada exclusivamente à Geometria e que, nos três últimos livros, trata da geometria esférica e da trigonometria, como ferramentas na Astronomia. Segundo Eves (2004, p.313), a Astronomia contribuiu tanto para a Matemática, que a designação de matemático significava, frequentemente, astrônomo. Nicolau Copérnico (1473-1543) percebeu que as hipóteses geocêntricas de Aristóteles e Ptolomeu não correspondiam à realidade e, contrariando também a Igreja, que colocava o homem no centro do Universo, revolucionou a visão do mundo ao defender a hipótese de um sistema heliocêntrico, segundo o qual a Terra se movia em torno do Sol. Em 1543, foi publicado o seu tratado "De Revolutionibus Orbitun Celestium", sobre as evoluções das órbitas celestes, com seções sobre Trigonometria.

A partir da primeira metade do século XVII, a introdução de novas notações algébricas, por Viète, fez com que a Trigonometria assumisse um moderno caráter analítico. $\mathrm{Na}$ sua obra "Cânon Mathemáticus", François Viète constrói tabelas de seis funções trigonométricas, com ângulos em aproximações de minutos. No lugar das frações sexagesimais, ele usou as decimais e desenvolveu métodos para resolver problemas de triângulos planos e esféricos, com essas seis funções.

No século XVII, o trabalho de matemáticos como Fermat e Descartes foi precursor das ideias do Cálculo Diferencial e Integral desenvolvidas por Newton e Leibniz. No século XVIII, Leonhard Euler (1707-1783), ao resolver problemas com equações diferenciais propostos por Daniel Bernoulli em 1735, desenvolveu seu método algébrico e, com a publicação do Introductio in analysin infinitorum em 1748, estabeleceu as bases da Análise, com o estudo de processos infinitos. Segundo Lima (2006, p.35), Euler inventou uma função que modernizou as noções de seno e cosseno, definida no triângulo retângulo, tendo por base a Análise Matemática recém criada, em consequência dos Cálculos Infinitesimais, o que permitiu substituir a medida do ângulo em graus pela medida em radianos, um número real qualquer $t$. Euler estabeleceu que o domínio de sua função $E$ é o conjunto IR dos números reais e o contradomínio é o círculo de raio unitário no plano cartesiano (que representamos por $S^{1}$ ). Os conteúdos explorados no ensino atual de Trigonometria, conforme encontramos em livros didáticos de Matemática, refazem o percurso histórico sobre o qual discorremos; no entanto, isso ocorre sem que se faça menção, em sala de aula, dos fatos históricos que acarretaram a evolução das razões no triângulo retângulo para as funções trigonométricas, perdendo-se, assim, uma ótima oportunidade para tornar as referências históricas uma fonte de motivação para os estudantes. 


\section{Referenciais teóricos}

\subsection{A teoria da Aprendizagem Significativa}

De acordo com essa teoria da psicologia educacional, na aquisição e retenção de novos conhecimentos, para que haja uma aprendizagem significativa, o material a ser aprendido deve ser logicamente significativo e, na estrutura cognitiva do aprendiz, devem existir conceitos capazes de "ancorar" esses novos conhecimentos, que os autores dessa teoria chamam de "subsunçores". Segundo Ausubel, Novak e Hanesian (1978), uma aprendizagem pode ser significativa ou mecânica. É significativa quando o conhecimento a ser aprendido interage com um determinado conceito presente na estrutura cognitiva do aprendiz (conceito subsunçor) e essa interação ocorre com a assimilação do novo conhecimento, por esse conceito subsunçor. Quando isso não acontece, a aprendizagem é mecânica, ou seja, "decorada" e sem nenhum apoio lógico, como os números de um telefone. Na aprendizagem escolar, esses autores classificam essa assimilação como recepção, quando o aluno recebe pronto todo o conteúdo a ser aprendido, ou seja, na sua forma final, ficando para este aluno a tarefa de internalizar esse conteúdo; e com a descoberta, quando o conteúdo a ser aprendido não é dado pronto, mas deve ser descoberto pelo aluno.

A maior parte das informações adquiridas pelos alunos, tanto dentro como fora da escola, é apresentada verbalmente e, sob o ponto de vista psicológico, a aprendizagem receptiva verbal é mais complexa, visto que exige um amadurecimento intelectual. Moreira (2006) observa que quando os conceitos e proposições são apresentados verbalmente, mas o aluno ainda não dispõe dos subsunçores necessários à aprendizagem significativa, ele pode aprender mecanicamente (ou automaticamente), até que alguns elementos de conhecimento presentes na sua estrutura cognitiva, ligados às novas informações, possam servir de subsunçores, mesmo que pouco elaborados. Nessa situação, Ausubel, Novak e Hanesian (1978, p.140) recomendam o emprego de organizadores prévios, um material introdutório que deve ser apresentado antes do material a ser aprendido, com as ideias mais abrangentes dos conhecimentos precedentes, ou até mesmo que possuam conceitos similares àqueles a serem aprendidos. É importante destacar que os organizadores não são necessariamente textos escritos, podendo ser uma discussão, uma demonstração, ou até mesmo um filme, dependendo das circunstâncias envolvidas na aprendizagem. Nesta pesquisa, durante os encontros com os alunos, planejamos, como organizadores prévios, atividades com materiais concretos e com o 
software Cabri-Géomètre II, para que os alunos pudessem redescobrir a invariância das razões trigonométricas em triângulos retângulos que são semelhantes.

Ao lembrar que, no processo da aprendizagem significativa, a nova informação deve interagir com os conceitos subsunçores do aprendiz, Moreira (2006) ressalta que esta situação reflete a "subordinação" entre o novo material e os conceitos subsunçores. Durante o processo de aprendizagem por subordinação, quando o conceito subsunçor sofre alguma modificação, podemos dizer que, com esse conceito está ocorrendo uma diferenciação progressiva; mas, quando a nova informação é aprendida por uma superordenação dos conceitos subsunçores, ou uma recombinação de ideias presentes na estrutura cognitiva, mas que não apresentam nenhuma dependência entre si, dizemos que, com esses conceitos, ocorreu uma reconciliação integrativa.

No nosso entendimento, de acordo com essa teoria, a aprendizagem significativa da função seno exige, em grande parte, a aplicação do princípio da reconciliação integrativa, com a combinação dos seguintes conceitos que devem estar presentes na estrutura cognitiva do aprendiz no momento da aprendizagem (conceitos subsunçores): relações trigonométricas no triângulo retângulo; medidas em radianos; ciclo trigonométrico; pontos no sistema cartesiano ortogonal; o conjunto dos números reais; domínio, contradomínio e imagem de uma função; e o Teorema de Pitágoras. Com a reconciliação integrativa, todos esses conceitos se recombinam, para darem sentido à definição do seno pela função de Euler $E: R \rightarrow S^{1}$.

As atividades, com propostas baseadas no uso de material concreto e de um software dinâmico, foram elaboradas para propiciar aos participantes um percurso por estes conceitos subsunçores, com o objetivo de tornar a aprendizagem da função seno significativa. Esta abordagem foi assim escolhida para permitir a passagem dos dados, inicialmente em forma de tabela, para a construção do gráfico da função seno. Em razão desta transição, adotamos como referencial teórico a Teoria dos Registros de Representação Semiótica.

\subsection{Registros de representação semiótica}

Na virada do milênio, na França, diante da complexidade do ambiente tecnológicocomputacional, surgiram as seguintes questões:

-Como podemos entender as dificuldades, frequentemente intransponíveis, que alguns estudantes têm na compreensão da Matemática?

- Qual a natureza e em quais aspectos identificamos estas dificuldades? 
Para Duval (2011), as respostas a estas perguntas não estão restritas a um ramo da Matemática ou à sua História, mas podem ser encontradas numa abordagem cognitiva que, segundo ele, pode determinar a origem dessa incompreensão e ajudar o estudante a entender e controlar a diversidade dos processos matemáticos. Segundo Duval (2011, Capítulo II, p. 40), sob o ponto de vista cognitivo, a Matemática se caracteriza pela grande variedade de representações semióticas como língua materna ${ }^{1}$, sistemas de numeração, figuras geométricas, escritas algébricas e formais, representações gráficas. A importância dessas representações baseia-se em dois aspectos: o acesso aos objetos matemáticos, que são abstratos; e a escolha de uma linguagem para a comunicação.

Sob o ponto de vista da aprendizagem e do ensino, na Matemática existem, segundo Duval (2011), dois tipos de transformações radicalmente diferentes: os tratamentos e as conversões. Os tratamentos são as transformações que acontecem dentro de um mesmo sistema de representação; por exemplo, durante um cálculo com números decimais ou na resolução algébrica de um sistema de equações, se permanecemos, respectivamente, nos números decimais ou na escrita algébrica do sistema de equações. As conversões são as transformações de um sistema de representação para outro; por exemplo, durante a passagem da escrita algébrica de uma função para o seu gráfico cartesiano.

De acordo com o tipo de representação e com o tipo de tratamento, as representações podem ser organizadas de acordo com o Quadro 1.

\begin{tabular}{|l|l|l|}
\hline & Representação discursiva & Representação não discursiva \\
\hline REGISTROS & Língua natural & Figuras geométricas planas \\
MULTIFUNCIONAIS: & Associações verbais & ou em perspectiva \\
Os tratamentos não & (conceituais). & (configurações nas dimensões 0, \\
são algoritmizáveis & Forma de raciocinar: & 1,2 ou 3) \\
& - Argumentação a partir de & -Apreensão operatória e não \\
& observações, de crenças... & somente perceptiva \\
& - Dedução válida a partir de & - Construção com instrumentos \\
\hline REGISTROS & definição ou teoremas. & \\
MONOFUNCIONAIS: & Sistemas de escritas: & - Numéricas (binária, decimal, \\
Os tratamentos são & fracionárias,...) & - Mudanças de sistemas de \\
principalmente & - Algébricas & - Interpolação, extrapolação \\
algoritmos & - Simbólicas (linguas formais) & \\
& - Cálculo & \\
\hline
\end{tabular}

Quadro 1 - Classificação dos diferentes tipos de registros semióticos no funcionamento matemático. Fonte: Duval, 2011, p. 118.

Duval (2011) também observa que os fracassos e os bloqueios, na compreensão dos alunos, ocorrem quando estes não conseguem reconhecer o mesmo objeto matemático em

\footnotetext{
${ }^{1}$ Neste texto, utilizamos língua materna para designar o uso da língua portuguesa na sala de aula de Matemática 
duas representações diferentes, pois defende que a compreensão em Matemática está intimamente ligada ao fato de um sujeito dispor de, ao menos, dois registros de representação diferentes, saber coordená-los e não confundir um objeto com a sua representação.

Para nós, são de especial importância os bloqueios de compreensão que eventualmente possam surgir, em razão dos tratamentos ou conversões entre registros semióticos utilizados com as funções trigonométricas, que são tabelas de valores e gráficos.

\section{Metodologia e procedimentos metodológicos}

Escolhemos a metodologia do Design Based Research, em razão de suas características retrospectivas e reflexivas. De acordo com Steffe e Thompson (2000), por volta de 1970, os pesquisadores em Educação Matemática perceberam que, para poderem entender a "matemática dos estudantes", primeiro era preciso desenvolver novos modelos de pesquisa com raízes na Educação Matemática, ao invés de simplificar e usar os modelos criados fora dela.

O primeiro artigo abordando o Design Based Research resumiu o pensamento de um grupo formado pelos pesquisadores Cobb, Confrey, Disessa, Lehrer e Schauble (2003), que tinham sólidos conhecimentos nas áreas das ciências cognitivas, psicologia, antropologia, inteligência artificial, biologia, matemática, da interação homem-computador. Em Cobb et al (2003), os autores ressaltam que a metodologia do design deveria possibilitar um contexto sujeito a testes e revisões sucessivas e iterativas, de forma sistemática, tendo como alvo um domínio específico da Matemática. Cobb et al (2003) relacionam algumas configurações e alvos dessa teoria e identificam algumas características do "design based experiments":

- uma nova teoria capaz de dar apoio ao processo de aprendizagem, não apenas para um aluno individualmente, mas para uma classe e até mesmo uma comunidade;

- de natureza intervencionista, possibilita uma melhoria educacional nessa nova forma de aprendizagem;

- a alternância de fases prospectivas e refletivas. Na fase prospectiva, as hipóteses são colocadas em prática e testadas em sala de aula e na fase retrospectiva, no caso de algumas dessas conjecturas serem rejeitadas, outras conjecturas alternativas poderão substituí-las em novos testes;

- a iteratividade dessa metodologia, formando um ciclo que termina quando todas as conjecturas forem testadas e revisadas. 
A flexibilidade desta metodologia de trabalho foi importante para a nossa pesquisa e, como relatamos a seguir, reformulamos o conjunto das atividades originalmente planejadas diante das avaliações a cada etapa de sua aplicação.

\subsection{O objetivo e o planejamento}

O objetivo de nossa pesquisa é verificar as contribuições de uma estratégia de ensino, formada pela combinação do contexto experimental com o contexto computacional para a aprendizagem significativa dos principais conceitos presentes na transição das razões para as funções trigonométricas. Promovemos um primeiro contato dos alunos com o software CabriGéomètre II, aplicamos um teste diagnóstico para identificar se os participantes estão familiarizados com os conceitos subsunçores necessários para a aprendizagem desses conceitos, realizamos quatro intervenções em oito encontros e, em cada intervenção, as atividades foram concluídas com uma ou duas questões avaliativas do conhecimento em jogo; e encerramos com um teste final. As intervenções foram conduzidas pelo terceiro autor, sob a orientação da primeira e a íntegra das atividades propostas encontra-se em Miashiro (2013). Em cada um dos encontros, foi apresentado um roteiro que orientou o trabalho dos alunos e a discussão do assunto escolhido. Os registros das atividades realizadas pelos alunos em papel e lápis e no computador foram utilizados para a análise. Com base na análise desses protocolos, obtidos ao longo das intervenções, foram realizadas as avaliações dos encontros, os ajustes necessários para o prosseguimento do trabalho e foram respondidas as questões de pesquisa.

\subsection{Participantes da pesquisa e a preparação do design inicial das quatro intervenções}

Os participantes desta pesquisa foram nove alunos de um curso noturno de uma universidade particular da cidade de São Paulo que cursavam o terceiro semestre de Licenciatura em Matemática. Devido ao reduzido número de participantes, abrimos mão do grupo de referência.

$\mathrm{Na}$ preparação do design inicial das quatro intervenções, levamos em conta os conceitos subsunçores identificados na análise do teste diagnóstico, assim como a ordem em que esses conceitos surgiram ao longo da História da Matemática.

Com foco na Trigonometria, analisamos: as recomendações contidas nos Parâmetros Curriculares Nacionais; as sugestões em pesquisas correlatas de Brighenti (1994), Lindegger (2000), Goios (2010); e as abordagens dadas aos conteúdos nos livros: "Trigonometria e 
Números Complexos" (CARMO; MORGADO; WAGNER, 2005); "Matemática, Contexto e Aplicações" (DANTE, 2009); Trigonometria (IEZZI, 2004); "Functions Modeling Change" (CONALLY; GLEASON; HUGHES-HALLET, 1998); e "Atividades com o Cabri-Géomètre II" (BALDIN; VILLAGRA, 2010).

\subsection{Os objetos do ambiente experimental}

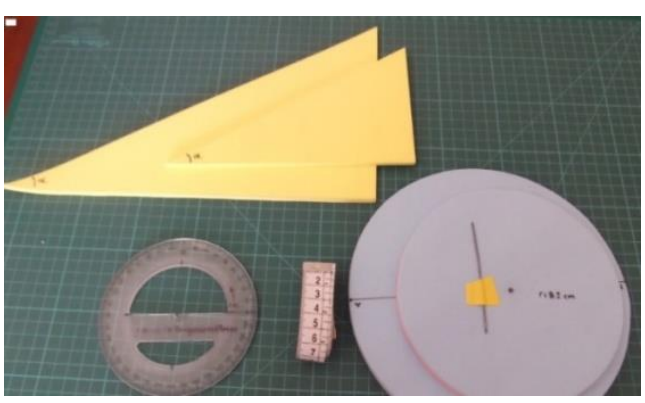

Figura 1 - Objetos do ambiente experimental Fonte: Nosso acervo (2013).

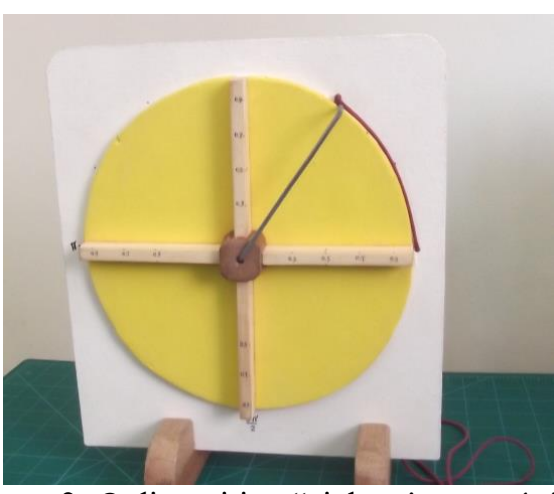

Figura 2- O dispositivo "ciclo-trigonométrico" Fonte: Nosso acervo (2013).

Para este ambiente, construímos, em EVA, um conjunto para cada participante com: três jogos com dois triângulos retângulos semelhantes; discos com raios distintos, cada um acompanhado de um arame com a medida do respectivo raio; um transferidor e uma fita métrica. Com os triângulos (ver Figura 1) foram propostas atividades envolvendo a semelhança entre os triângulos e as relações trigonométricas; com o disco e o arame, as medidas em radianos. Construímos também o dispositivo "ciclo trigonométrico" (ver Figura 2) que o pesquisador utilizou para explicar as características do objeto matemático que leva esse nome.

\subsection{O programa Cabri-Géomètre II e o ambiente computacional}

O Cabri-Géomètre (abreviação da palavra francesa "cahier de brouillon intéractif") é um software interativo que possui ferramentas com as quais é possível construir figuras dinâmicas na tela do computador, substituindo o lápis, o papel, a régua e o compasso. Essas figuras podem ser deslocadas, ampliadas ou reduzidas, mas continuam preservando suas propriedades, se construídas adequadamente. Com as ferramentas do Cabri-Géomètre II, podemos dispor dos eixos cartesianos, medir o comprimento de segmentos e arcos, medir ângulos, transferir o comprimento de um segmento para outro e até mesmo transferir o comprimento de um arco para um segmento. As construções dinâmicas desse software podem 
facilitar o estudo dos teoremas da geometria euclidiana plana e da Trigonometria. Suas construções podem ser salvas em arquivos e revistas passo a passo. Todos esses recursos transformaram o programa computacional Cabri-Géomètre numa valiosa ferramenta educacional. Durante nossas intervenções com os alunos, em cada assunto, sempre que possível, procuramos alternar o material concreto e o software Cabri-Géomètre, numa tentativa de generalizar as conjecturas feitas com o material concreto.

\subsection{O teste diagnóstico e as intervenções}

O teste diagnóstico teve por objetivo identificar os conhecimentos dos participantes sobre a trigonometria do triângulo retângulo, o teorema de Pitágoras e suas aplicações, as medidas de ângulos (em graus e radianos) e a definição da função seno. Constatou-se que os alunos, em geral, não dominam estes conhecimentos em razão da pouca compreensão sobre a semelhança de triângulos. Esta constatação orientou o planejamento das intervenções nos ambientes experimental e computacional, de forma a iniciar as atividades a partir da discussão sobre as propriedades de semelhança e suas aplicações.

A primeira intervenção teve como objetivo explorar as razões trigonométricas (seno, cosseno e tangente) no triângulo retângulo, com o material concreto e com o Cabri-Géomètre. A partir dessa exploração, os alunos construíram uma tabela para os senos dos ângulos de medidas $30^{\circ}, 45^{\circ}$ e $60^{\circ}$.

O objetivo da segunda intervenção foi promover situações com o Cabri-GéomètreII e os materiais concretos, para mostrar que as medidas dos ângulos, em radianos, independem da medida do raio da circunferência.

A terceira intervenção teve como objetivos criar um organizador prévio para a função seno, com a contextualização de uma situação para identificar e estudar um fenômeno periódico e apresentar a definição do ciclo trigonométrico.

O objetivo da quarta intervenção foi o estudo da definição da função seno e a construção de gráficos dessa função, no papel e com o Cabri-Géomètre II.

A cada encontro, os pesquisadores avaliaram o trabalho dos participantes e analisaram seus protocolos no sentido de ajustar as atividades de forma a promover um melhor aproveitamento e compreensão dos conteúdos em discussão.

O teste final retomou os vários aspectos dos conteúdos já apresentados para o teste inicial, quais sejam, das relações trigonométricas no triângulo retângulo até a construção da função seno. As atividades e testes podem ser encontrados, na íntegra, em Miashiro (2011). 
Neste texto, ressaltamos apenas alguns trechos da terceira e da quarta intervenções, nas quais verificamos os melhores resultados da aplicação de nossa estratégia de ensino, formada pela combinação dos contextos materiais e computacionais.

Na terceira intervenção, apresentamos o dispositivo "ciclo trigonométrico" (ver Figura 2), como uma extensão da lousa e do giz. Falamos sobre as medidas em radianos, mostramos o cordão que representa um arco, os eixos cartesianos, materializados pelas réguas cruzadas, o círculo orientado e a primeira determinação de um arco. Nessa ocasião, ouvimos o comentário de um aluno, afirmando que, a partir daquele instante, passava a entender a medida 3,14 rd. Em seguida explicamos, com o Cabri e um projetor de imagens, os passos para a construção de um círculo trigonométrico. Com essa figura, reproduzida no Quadro 2, repetimos as explicações que havíamos feito anteriormente e ativamos a ferramenta "coordenadas", para um ponto colocado nesse círculo. Com esse ponto na figura dinâmica, os alunos seguiram as instruções da atividade, para calcular a soma dos quadrados dessas coordenadas e puderam perceber a genialidade de Euler, ao criar o círculo trigonométrico para definir a sua função $E(x)$, de um número real $x$.

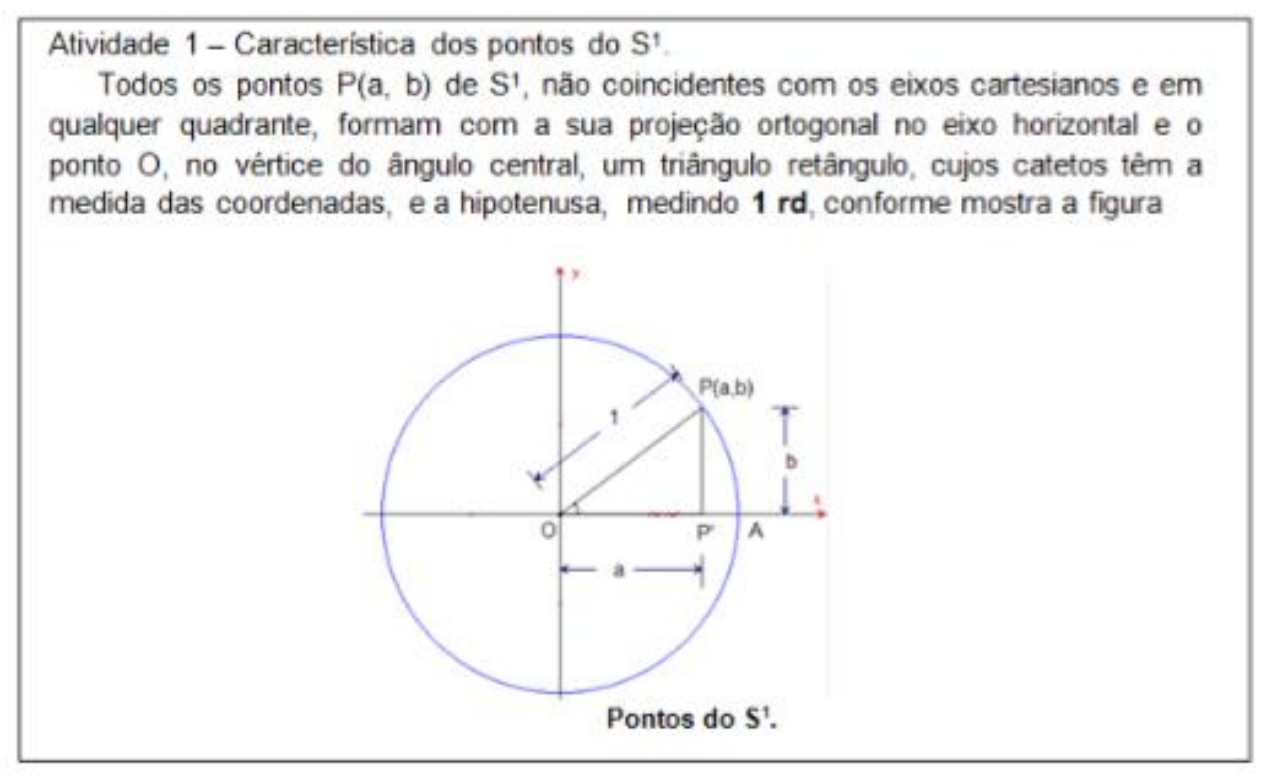

Quadro 2 - $3^{\mathrm{a}}$ intervenção, atividade 1.

Fonte: Nosso acervo (2013).

Na Figura 3, exibimos a participação do aluno 3 nessa atividade; verificações semelhantes foram feitas pelos demais participantes presentes, o que mostra que conseguiram observar corretamente a identidade trigonométrica em estudo. 


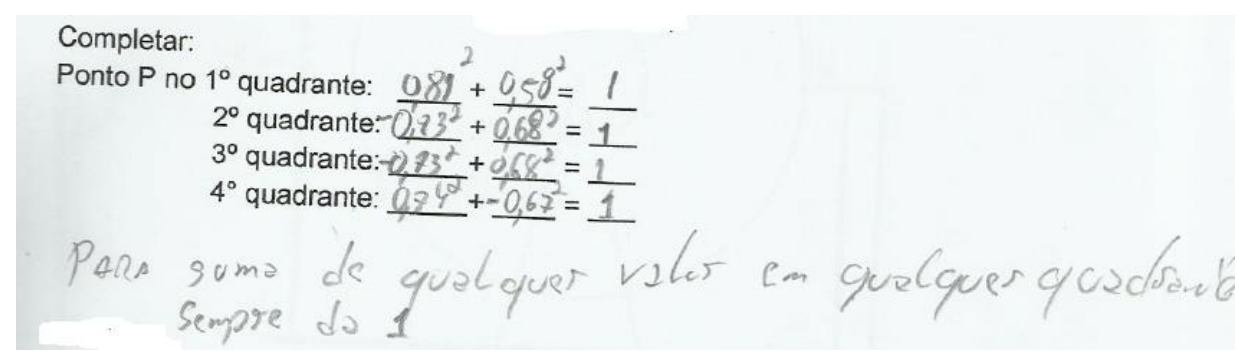

Figura 3- Aluno 3, $3^{\text {a }}$ Intervenção, atividade 1.

Fonte: Nosso acervo (2013).

Para iniciar a próxima atividade, pedimos que fizessem a leitura de um texto com a definição de uma função periódica e passamos à contextualização da "roda-gigante". Para esta contextualização, apresentamos o arquivo que contém uma representação da dinâmica do movimento da roda gigante, explicamos como funciona essa dinâmica e pedimos aos participantes que utilizassem o arquivo pronto para determinar os registros do altímetro com o movimento de um ponto da roda (ver Figura 4).

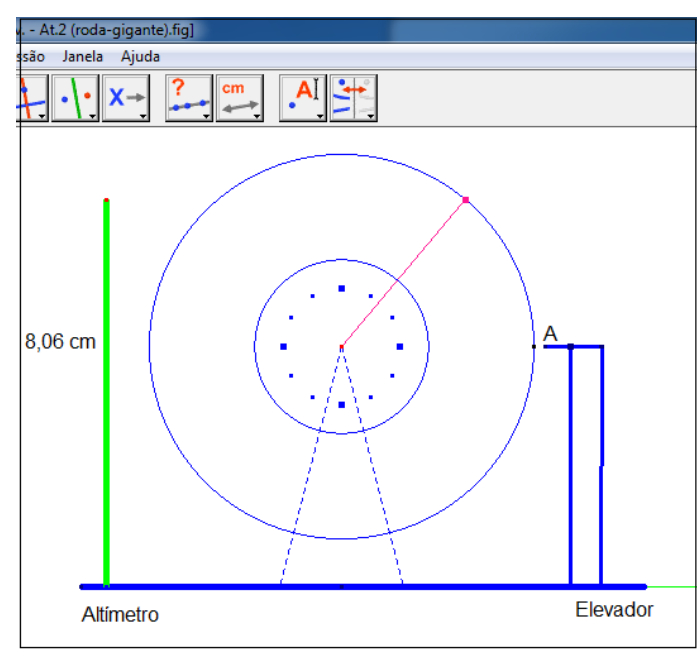

Figura 4 - $3^{\text {a }}$ Intervenção, atividade 2 Fonte: Nosso acervo (2013).

$\mathrm{Na}$ Figura 4, do lado esquerdo, representamos um marcador da altura da gôndola representada na figura pelo ponto $P$. Com o "ponteiro", é possível deslocar a "gôndola" nessa figura dinâmica. No texto dessa atividade, colocamos a orientação:

Você terá que embarcar como passageiro numa roda-gigante que dispõe de um elevador para deixá-lo na plataforma de embarque, no ponto A da figura. Esta roda, que está representada em escala na figura, gira em sentido anti-horário e o diâmetro de sua circunferência, na representação, é de $8 \mathrm{~cm}$. A altura da plataforma A (a altura do centro da roda), na representação, é de $5 \mathrm{~cm}$. (Fonte: própria.)

Informamos que essa roda demora uma hora para dar uma volta completa, e a tarefa dos alunos consiste em preencher uma tabela com a altura do passageiro a cada cinco minutos e, com os dados dessa tabela, construir um gráfico. 
Na Figura 5, exibimos a tabela preenchida pelo aluno 7, seguida do gráfico de uma função ainda não identificada. Todos os quatro alunos realizaram com sucesso esta atividade.

\begin{tabular}{|c|c|c|c|c|c|c|c|c|c|c|c|c|c|}
\hline$t(\min )$ & 0 & 5 & 10 & 15 & 20 & 25 & 30 & 35 & 40 & 45 & 50 & 55 & 60 \\
\hline$h(\mathrm{t})(\mathrm{cm})$ & 5 & 7 & 8,5 & 9 & 8,5 & 7 & 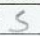 & 3 & 1 & 15 & 13 & 3 & 5 \\
\hline \multicolumn{14}{|c|}{ Tabela 2 - Altura do passageiro em relação } \\
\hline$t$ (min) & 65 & 70 & 75 & 80 & 85 & 9 & & & 100 & 105 & 110 & 115 & 120 \\
\hline$h(t)(c m)$ & 7 & 85 & 9. & 8.5 & 7 & 1 & & 3 & 15 & 1 & 1,5 & 3 & 5 \\
\hline
\end{tabular}

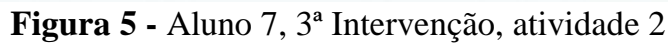
Fonte: Nosso acervo (2013).

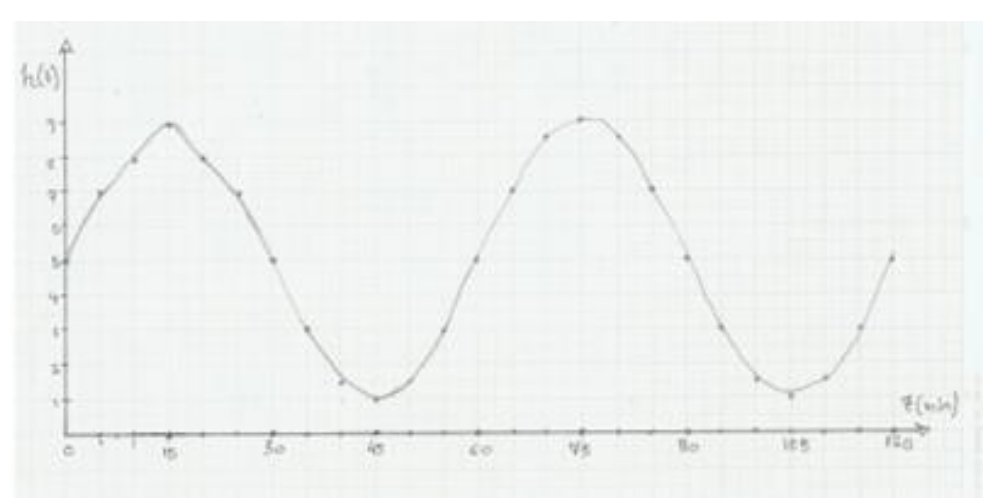

Figura 6 -Aluno $7,3^{\text {a }}$ Intervenção, atividade 2 Fonte: Nosso acervo (2013).

Na quarta intervenção, que teve como objetivo o estudo da função seno, a sequência de atividades foi semelhante à anterior, ou seja, os alunos trabalharam com uma figura do Cabri (neste caso puderam construir) que, manipulada, forneceu as coordenadas (ferramenta do Cabri) relativas ao valor do seno, em intervalos de $30^{\circ}$ e com esses valores construíram o gráfico da função seno. Dos seis alunos que participaram, todos preencheram corretamente a tabela trigonométrica, mas apenas uma dupla, a formada pelos alunos 4 e 7 , conseguiu traçar o gráfico da função seno.

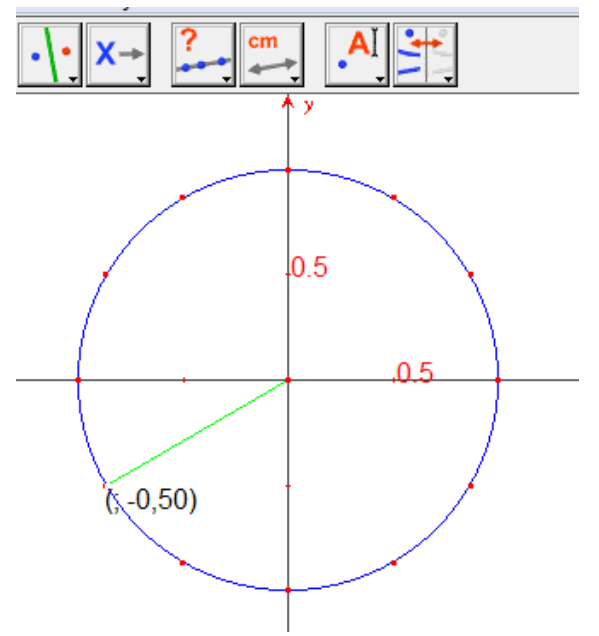

Figura 7-Tela do aluno 8 , no ângulo de $210^{\circ}$ Fonte: Nosso acervo (2013).

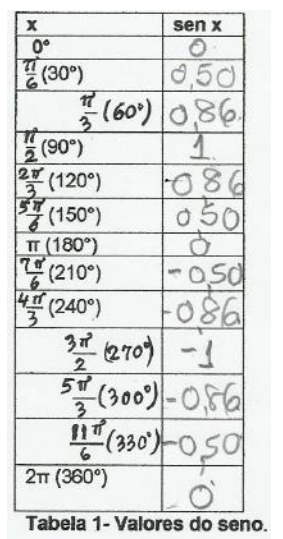

Figura 8 - Tabela do aluno 8 Fonte: Nosso acervo (2013). 


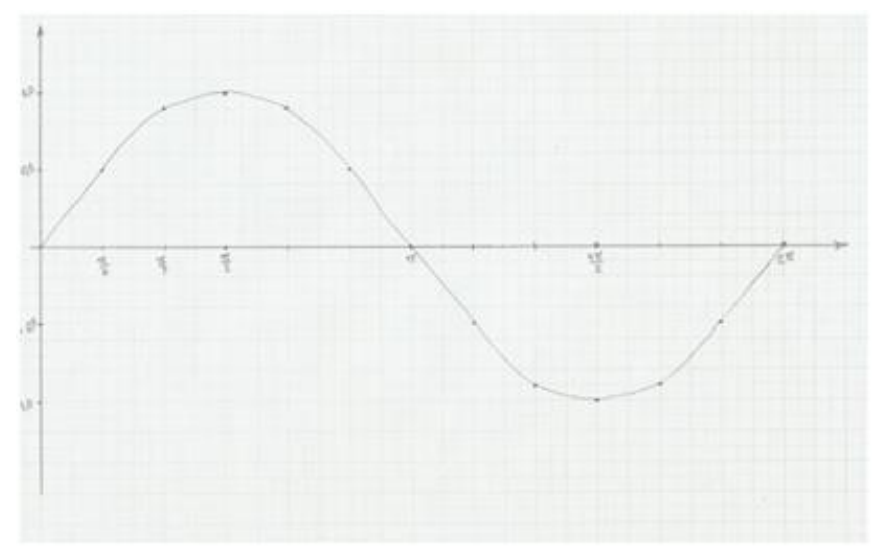

Figura 9 - Alunos 4 e 7, gráfico da função seno Fonte: Nosso acervo (2013).

De acordo com a teoria cognitiva dos registros de representação semiótica de Duval, a conversão de registros, como temos nesta atividade, se constitui numa das dificuldades para a aprendizagem de Matemática e "a compreensão em matemática implica a capacidade de mudar de registro" (DUVAL, 2008, p. 21). É importante lembrar que o teste diagnóstico indicou que nenhum desses alunos sabia como traçar o gráfico da função seno e os resultados das intervenções que aplicamos nos fazem otimistas quanto ao potencial didático da estratégia de ensino utilizada, uma vez que, na terceira atividade, as representações e a conversão do ciclo trigonométrico para a tabela foi realizada por todos os seis participantes. $\mathrm{O}$ mesmo não ocorreu na quarta atividade, na qual a conversão entre os dados da tabela e o gráfico da função seno só foi feita adequadamente por uma das três duplas presentes; as outras duas fizeram o gráfico de uma função periódica que não corresponde aos dados da tabela.

\section{Considerações finais}

Constatamos que a aplicação da "estratégia de ensino", formada pela combinação do contexto experimental com o contexto computacional, com os alunos construindo as figuras dinâmicas do Cabri Géomètre II, trouxe contribuições para a aprendizagem significativa dos conceitos subsunçores da trigonometria, na aprendizagem das medidas dos ângulos em radianos, na construção de tabelas trigonométricas e do gráfico de uma função periódica. Esta combinação, juntamente com a avaliação constante das estratégias adotadas ao longo das atividades, possibilitou explorar os vários aspectos dos conteúdos considerados como subsunçores para a construção de uma função trigonométrica. Desta forma, conseguimos atingir parte dos objetivos e responder a nossa questão de pesquisa. O comparecimento irregular às atividades e as dificuldades dos nove alunos em fundamentos de Matemática, relativas aos conteúdos da Educação Básica, identificados no teste diagnóstico, certamente 
interferiram nos resultados desta pesquisa e, embora todos os seis participantes que vieram à última atividade tenham conseguido montar uma tabela correta com valores da função seno, quatro deles esboçaram o gráfico de uma função periódica, mas que não corresponde aos valores dessa tabela e apenas uma dupla fez um esboço correto da função seno, como era o esperado ao final de nossa intervenção.

Sabemos que o estudo das razões trigonométricas é uma parte de um conjunto de conceitos subsunçores da trigonometria e consideramos que o conceito mais complexo desse grupo é o do ciclo trigonométrico, cuja aprendizagem significativa depende do princípio da reconciliação integrativa (AUSUBEL; NOVAK; HANESIAN, 1978), para que haja a combinação dos seguintes conceitos: sistema cartesiano ortogonal, definição de lugar geométrico do círculo, arco orientado, as medidas em radianos e o conjunto dos números reais. Ressaltamos que esses conceitos são constituídos por ideias paralelas, que não guardam entre si uma dependência sequencial e, nessa situação, de acordo com Ausubel, Novak e Hanesian (1978, p.161), compreender parte dos conceitos não pressupõe a compreensão do conjunto.

Para futuras pesquisas, tanto no ensino como na aprendizagem de Trigonometria, nossa sugestão é que se persista na combinação dos contextos materiais e computacionais e, sempre que possível, se recorra à História da Matemática. Esperamos que este trabalho possa contribuir para a Educação Matemática e sirva de inspiração para novos pesquisadores.

\section{Referências}

AABOE, A. Episódios da História Antiga da Matemática. Rio de Janeiro: Editora SBM, 1997.

AUSUBEL, D.; NOVAK, D. J.; HANESIAN, H. Psicologia Educacional. Tradução de E. Nick; H. B. Rodrigues; L. Peotta; M. A. Fontes e R. M. Maron. São Paulo: Edgard Blucher, 1978.

BALDIN, Y.Y; VILLAGRA, G.A.L. Atividades com Cabri-Géomètre II. São Carlos: EdUFSCar, 2007.

BOYER, C. C. História da Matemática. São Paulo: Edgard Blucher, 1996.

BRIGHENTI, M. J. L. Ensino e aprendizagem da trigonometria: novas perspectivas da Educação Matemática. 1994. 174 p. Dissertação (Mestrado em Educação Matemática) - Universidade Estadual Paulista, Rio Claro, 1994.

CARMO, M. P.; MORGADO, A. C.; WAGNER, E. Trigonometria e Números Complexos. Rio de Janeiro: Editora SBM, 2005.

COBB, P. et al. Design experiments in educational research. Educational Researcher, Washington, DC, n. 32 (1), p. 9-13, 2001. 
CONNALlY, E.; GLEASON, A. M.; HUGHES-HALLET, D. Functions Modeling Change: a preparation for Calculus. New York: John Wiley \& Sons, 1998.

DANTE, L. R. Matemática Contexto \& Aplicações. São Paulo: Ática, 2009.

DUVAL, R. A. cognitive analysis of problems of comprehension in a learning of mathematics.

Educational Studies in Mathematics, Berlim, v. 61, n. 1/2, p. 103-131. 2006.

DUVAL, R. Aprendizagem em matemática - registros de representação semiótica. In: MACHADO, A. S. Educação Matemática - uma (nova) introdução. São Paulo: Papirus, 2008. p. 11-33.

DUVAL, R. Semiósis e Pensamento Humano - registros semióticos e aprendizagens intelectuais. Tradução de R. A. Silveira e F. L. Levy. São Paulo: Livraria da Física Editora, 2009.

DUVAL, R. Ver e ensinar a matemática de outra forma - entrar no modo matemático de pensar: os registros de representação semiótica. Tradução de M. A. Dias. São Paulo: Editora Proem, 2011.

EVES, H. Introdução à história da Matemática. Tradução de H. H. Domingues. Campinas: Editora Unicamp, 2004.

GOIOS, D. F. Potencialidades didático-pedagógicas de um objeto para a aprendizagem: uma análise através da Teoria da Cognição Corporificada para o ensino de Trigonometria. 2010. 134 p. Dissertação (Mestrado em Educação Matemática) - Universidade Bandeirante de São Paulo, São Paulo, 2010.

IEZZI, G. Trigonometria. São Paulo: Atual, 2004. (Fundamentos da Matemática Elementar, 3).

LIMA, E. L. Meu professor de Matemática e outras histórias. Rio de Janeiro: Editora SBM, 2006.

KATZ, V. J. A History of Mathematics - an introduction. Boston: Addison-Wesley, 2009.

LINDEGGER, L. R. Construindo os conceitos básicos da trigonometria no triângulo retângulo: uma proposta a partir da manipulação de modelos. 2000. 106 f. Dissertação (Mestrado em Educação Matemática) - Faculdade de Ciências Exatas e Tecnologias, Pontifícia Universidade Católica, São Paulo, 2000.

LOBO DA COSTA, N. M. Funções seno e cosseno: uma sequência de ensino a partir dos contextos do mundo experimental e do computador. 1997. 125 f. Dissertação (Mestrado em Ensino da Matemática) - Faculdade de Ciências Exatas e Tecnologias, Pontifícia Universidade Católica, São Paulo. 1997.

MAOR, E. Trigonometric Delights. New York: Princeton University Press, 1998.

MIASHIRO, P. M. A transição das razões para as funções trigonométricas. 2013. 97 f. Dissertação (Mestrado em Educação Matemática) - Universidade Bandeirante de São Paulo, São Paulo, 2013.

MOREIRA, M. A. A teoria da aprendizagem significativa e sua implementação em sala de aula. Brasília: Editora da UnB, 2006.

STEFFE, L. P.; THOMPSON, P. W. Teaching experiment methodology: underling principles and essential elements. In: LESH, R.; KELLY, A. E. (Ed.). Research design in mathematics and Science education. Hillsdale: Erlbaum, 2000. p. 267-307.

YAMAMOTO, Y. B.; VILLAGRA, G. A. Atividades com Cabri-Géomètre II. São Carlos: EdufsCar, 2010. 
Submetido em Junho de 2015. Aprovado em Outubro de 2015. 